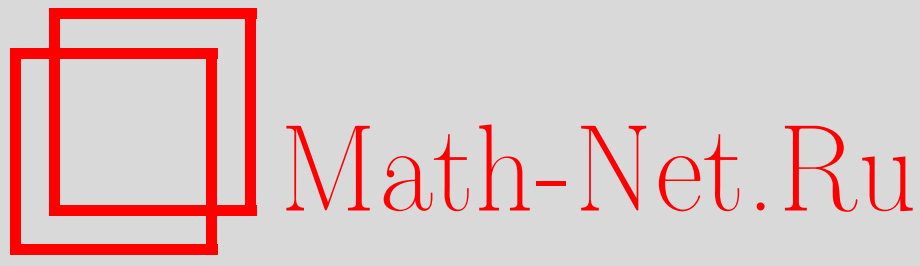

Обцероссийский математический портал

Е. В. Жужома, О непрерывной зависимости геодезических каркасов в хаусдорфовой метрике, Матем. заметки, 2005, том 77, выпуск 6, 935-937

DOI: https://doi.org/10.4213/mzm2554

Использование Общероссийского математического портала Math-Net.Ru подразумевает, что вы прочитали и согласны с пользовательским соглашением http://www.mathnet.ru/rus/agreement

Параметры загрузки:

IP : 54.205 .225 .156

26 апреля 2023 г., $17: 12: 27$ 


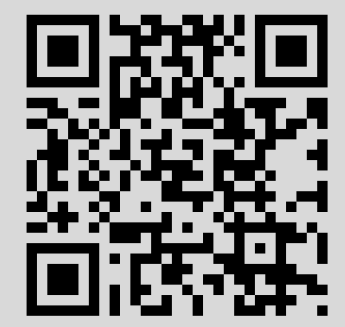




\section{О НЕПРЕРЫВНОЙ ЗАВИСИМОСТИ ГЕОДЕЗИЧЕСКИХ КАРКАСОВ В ХАУСДОРФОВОЙ МЕТРИКЕ}

\section{Е.В. Жужома}

\section{Статья посвящается моему учителю С.X. Арансону в честь его 70-летия}

Важным топологическим инвариантом потоков на двумерном торе $T^{2}$ (ориентируемой замкнутой поверхности рода 1) является число вращения Пуанкаре, которое отражает асимптотическое поведение траекторий потока. Для минимальных потоков на торе число вращения является полным топологическим инвариантом (с точностью до пересчета с помощью автоморфизма фундаментальной группы), иррационально и непрерывно зависит от возмущения векторного поля, порождающего поток, в $C^{1}$ топологии пространства векторных полей на $T^{2}$.

Любой поток на ориентируемой замкнутой поверхности $M^{2}$ рода $\geqslant 2$ имеет точки покоя и, следовательно, не является минимальным. Аналогом минимального потока на $M^{2}$ является cверxтранзитивный поток, любая одномерная траектория которого всюду плотна на поверхности. Сверхтранзитивный поток, вообще говоря, может иметь любое число точек покоя нулевого индекса (так называемые фальшивые седла), которые не связаны с топологией поверхности. Сверхтранзитивный поток без точек покоя нулевого индекса назьвается иррачиональным.

В 60-х годах 20-го века Д. В. Аносовым была сформулирована концепция о том, что основным методом исследования нетривиальных инвариантных множеств в нелокальной теории динамических систем и слоений на поверхностях является изучение асимптотики поднятий траекторий и слоев на универсальню накрывающую плоскость с помощью абсолюта этой плоскости. Развитие этих идей привело к топологической классификации основных классов потоков, слоений и гомеоморфизмов с инвариантньми слоениями на замкнутых поверхностях, а также к решению ряда важных проблем в качественной теории динамических систем на $n$-мерных многообразиях (более подробно об этом см. в [1], [2]).

Пусть $M^{2}$ наделена структурой риманова многообразия постоянной отрицательной кривизны. Для иррациональных потоков на $M^{2}$ полньм топологическим инвариантом (с точностью до пересчета с помощью автоморфизма фундаментальной группы) является гомотопический класс вращения, введенный С. Х. Арансоном и В.З. Гринесом [3], который можно представить в виде специалњной геодезической ламинации (геодезического каркаса потока), состоящей из геодезических с

Работа выполнена при поддержке Российского фонда фундаментальных исследований, гранты № 02-01-000-98 и № 05-01-00501. 
теми же самыми асимптотическими направлениями, что и траектории потока [2]-[5]. Такое представление более удобно, поскольку множество геодезических ламинаций наделяется естественной структурой топологического пространства, и можно рассмотреть задачу о зависимости геодезического каркаса от возмушения потока (или от параметра для однопараметрического семейства потоков). Эту задачу можно рассмотреть также для геодезических каркасов слоений.

Геодезический каркас иррационального потока или слоения является минимальной неприводимой геодезической ламинацией, состоящей из незамкнутых нетривиально рекуррентных геодезических [6]-[9]. Такой каркас естественно назвать иррациональным. В некотором смысле, иррациональный геодезический каркас является аналогом числа вращения Пуанкаре минимального потока на торе. Геодезический каркас назьвается рачиональным, если он не содержит нетривиально рекуррентных геодезических. Отметим, что рациональный геодезический каркас содержит замкнутые геодезические. Обозначим через $\mathscr{L}_{\text {or }}\left(M^{2}\right)$ множество ориентируемых геодезических ламинаций на $M^{2}$.

Для определения метрики на $\mathscr{L}_{\text {or }}\left(M^{2}\right)$ мы будем использовать хаусдорфову метрику на пространстве замкнутых подмножеств касательного расслоения $T\left(M^{2}\right)$. Пусть $d(\cdot, \cdot)$ - метрика постоянной отрицательной кривизны на поверхности $M^{2}$. Эта метрика индуцирует метрику $\hat{d}(\cdot, \cdot)$ на $T\left(M^{2}\right)$. Напомним, что для данных замкнутых множеств $A, B \subset T\left(M^{2}\right)$ xаусдорфово расстояние между $A$ и $B$ определяется следующим образом:

$$
\hat{d}_{H}(A, B)=\max \left\{\max _{x \in A} \min _{y \in B} \hat{d}(x, y), \max _{x \in B} \min _{y \in A} \hat{d}(x, y)\right\} .
$$

Нетрудно проверить, что $\hat{d}_{H}(A, B)<\varepsilon$, если и только если $A \subset N_{\varepsilon}(B)$ и $B \subset N_{\varepsilon}(A)$, где $N_{\varepsilon}(C)$ означает $\varepsilon$-окрестность множества $C$.

Для геодезической ламинации $\lambda \subset \mathscr{L}_{\text {or }}\left(M^{2}\right)$ обозначим через $\hat{\lambda}$ ее поднятие в $T\left(M^{2}\right)$. Из определения геодезической ламинации вытекает, что $\hat{\lambda}$-замкнутое множество. Мы определим хаусдорфово расстояние между геодезическими ламиначиями $\lambda, \lambda^{\prime} \subset \mathscr{L}_{\text {or }}\left(M^{2}\right)$ как

$$
d_{H}\left(\lambda, \lambda^{\prime}\right) \stackrel{\text { def }}{=} \hat{d}_{H}\left(\hat{\lambda}, \hat{\lambda}^{\prime}\right) .
$$

Множество $\hat{\lambda}$ является минимальньм множеством геодезического потока на $M^{2}$. Поэтому введенное расстояние между геодезическими ламинациями можно рассматривать как классическое хаусдорфово расстояние между соответствующими минимальными множествами геодезического потока.

Поскольку, как указьвалось ранее, любой поток на $M^{2}$ имеет точки покоя, то естественно (учитывая характер решаемой задачи) ограничиться потоками с грубыми точками покоя. Обозначим через $\chi^{1}\left(M^{2}\right)$ пространство $C^{1}$ векторных полей на $M^{2}$, наделенное $C^{1}$ топологией, и через $\tilde{\chi}^{1}\left(M^{2}\right) \subset \chi^{1}\left(M^{2}\right)$ - векторные поля только с грубыми состояниями равновесия. Для потока $f^{t}$ обозначим через $G\left(f^{t}\right)$ его геодезический каркас. Ясно, что для $f^{t}$ с конечным числом точек покоя $G\left(f^{t}\right) \subset \mathscr{L}_{\text {or }}\left(M^{2}\right)$. Имеет место следующая

ТЕОРЕма 1. Пусть $f^{t}$ - иррациональный поток на замкнутой ориентируемой поверхности $M^{2}$ рода $\geqslant 2$, порождаемый векторным полем $v \in \widetilde{\chi}^{1}\left(M^{2}\right)$, и пусть $U$ - окрестность геодезического каркаса $G\left(f^{t}\right)$. Тогда существует окрестность $N(v) \subset \chi^{1}\left(M^{2}\right)$ векторного поля $v$ такая, что

1) любое векторное поле $w \in N(v)$ порохдает поток $g^{t}$ с непустым геодезическим каркасом $G\left(g^{t}\right) \subset \mathscr{L}_{\mathrm{or}}\left(M^{2}\right)$ таким, ито $G\left(g^{t}\right) \in U$

2) для любой окрестности $N^{\prime} \subset N(v)$ существует векторное поле $w \in N^{\prime}$ такое, что у потока $g^{t}$, индуиированного полем $w$, геодезический каркас $G\left(g^{t}\right)$ рачиональный.

Таким образом, геодезический каркас иррационального потока с грубыми точками покоя обладает такими же свойствами, что и иррациональное число вращения Пуанкаре минимального потока на торе:

1) непрерьвность (в метрике Хаусдорфа) относительно возмушения потока, 
2) “нестабильность”, т.е. сколь угодно малым возмущением исходный иррациональный каркас может быть превращен в рациональный каркас.

Аналогичные определения вводятся для слоений, только вместо пространства ориентируемых геодезических ламинаций $\mathscr{L}_{\text {or }}\left(M^{2}\right)$ рассматривается пространство ламинаций $\mathscr{L}\left(M^{2}\right)$, составленных из ненаправленных геодезических. Для определения метрики на $\mathscr{L}\left(M^{2}\right)$ используется проективное касательное расслоение $P T\left(M^{2}\right)$, составленное из пар $(x, l)$, где $x \in M^{2}$ и $l$ - прямая, проходящая в касательном пространстве $T_{x}\left(M^{2}\right)$ через начало координат [7], [8]. Через $\mathscr{F}^{1}\left(M^{2}\right)$ обозначается пространство $C^{1}$ слоений на $M^{2}$. Имеет место следующая

ТЕОрема 2. Пусть $F$ - иррачиональное $C^{1}$ слоение на замкнутой ориентируемой поверхности $M^{2}$ рода $\geqslant 2$ с грубими особенностями седлового типа (отрицательного индекса) и $U$ - окрестность геодезического каркаса $G(F)$ слоения $F$. Тогда существует окрестность $N(F)$ слоения $F$ в пространстве $\mathscr{F}^{1}\left(M^{2}\right)$ такая, что

1) любое $C^{1}$ слоение $H \in N(F)$ имеет непустой геодезический каркас $G(H)$, который принадлехсти $U$;

2) для любой окрестности $N^{\prime} \subset N(F)$ существует $C^{1}$ слоение $H \in N^{\prime}$ такое, что геодезический каркас $G(H)$ рачиональный.

Отметим, что имеется принципиальное отличие между полными инвариантами потоков (слоений) на торе $T^{2}$ и на поверхности $M^{2}$ рода $\geqslant 2$. Всевозможные числа вращения заполняют всю числовую прямую, а метрическое пространство $\left(\mathscr{L}\left(M^{2}\right), \hat{d}_{H}\right)$ вполне разрьвное и имеет нулевую хаусдорфову размерность [7].

Автор благодарит Д. В. Аносова, С. Х. Арансона и В. С. Медведева за плодотворные обсуждения.

\section{СПИСОК ЦИТИРОВАННОЙ ЛИТЕРАТУРЫ}

1. Аносов Д. В. // Изв. АН СССР. Сер. матем. 1987. Т. 51. №1. С. 16-43. 2. Аносов Д. В., Ж ужжома Е. В. // Тр. МИАН. 2002. Т. 238. С. 5-54. 3. Арансон С. Х., Гринес В. 3. // Матем. сб. 1973. Т. 90. № 3. С. 372-402. 4. Арансон С. Х., ЖКужома Е. В., Медведев В. С. // Матем. сб. 1997. Т. 188. № 7. С. 3-22. 5. Арансон С. Х., ЖКужома Е. В., Медведев В. С. // Докл. РАН. 1998. T. 362. № 1. C. 7-11. 6. Aranson S. Kh., Belitsky G. R., Zhuzhoma E. V. Introduction to the Qualitative Theory of Dynamical Systems on Surfaces. Transl. Math. Monographs. V. 153. Providence, RI: Amer. Math. Soc., 1996. 7. Bonahon F., Zhu X. // Geometry and Topology. 2004. V. 8. P. 539-564. 8. Casson A. J., Bleiler S. A. Automorphisms of Surfaces after Nielsen and Thurston. London Math. Soc. Student Texts. Cambridge: Cambridge Univ. Press, 1988. 9. Nikolaev I., Zhuzhoma E. Flows on 2-Dimensional Manifolds. An Overview. Lecture Notes in Math. V. 1705. Berlin: Springer-Verlag, 1999. 